\title{
ATITUDES DOS ENFERMEIROS ESPECIALISTAS FACE À MORTE: PARTICULARIDADES DOS ENFERMEIROS ESPECIALISTAS EM ENFERMAGEM DE REABILITAÇÃO
}

ACTITUDES DE ENFERMERAS ESPECIALIZADAS HACIA LA MUERTE: PARTICULARIDADES DE LAS ENFERMERAS ESPECIALIZADAS EN ENFERMERÍA DE REHABILITACIÓN

\section{ATTITUDES OF SPECIALIST NURSES TOWARDS DEATH: PARTICULARITIES OF SPECIALIST NURSES IN REHABILITATION NURSING}

DOI 10.33194/rper.2020.v3.n1.10.5772 | Submetido 05/05/2020 | Aprovado 15/09/2020

\section{Maria Filomena Passos Teixeira Cardoso, 2,3 $\mathbb{D}$; Maria Manuela Ferreira Pereira Da Silva Martins ${ }^{4,5} \mathbb{D}$; Olga Maria Pimenta Lopes Ribeiro ${ }^{4,5}$; ; Esmeralda Faria Fonseca ${ }^{2}$ (i)}

1 - Universidade do Porto (UP), Instituto de Ciências Biomédicas Abel Salazar; 2 - Centro Hospitalar Universitário São João; 3 - Universidade Fernando Pessoa; 4 - Escola Superior de Enfermagem do Porto;

5 - Centro de Investigação em Tecnologias e Serviços de Saúde

\section{RESUMO}

Objetivo: analisar as diferenças no perfil de atitudes face à morte entre enfermeiros especialistas em enfermagem de reabilitação e enfermeiros especialistas em outras áreas.

Método: estudo comparativo, quantitativo, transversal, com participação de 223 enfermeiros especialistas de um hospital do norte de Portugal. Como instrumento de colheita de dados usou-se o questionário. Para análise dos dados recorreu-se a estatística descritiva e analítica.

Resultados: quanto às atitudes face à morte, nos enfermeiros especialistas em enfermagem de reabilitação predominaram as de aproximação, neutralidade, medo, evitamento e escape. Nos enfermeiros com especialização em outras áreas prevaleceram as atitudes de aproximação, medo, neutralidade, evitamento e escape.

Conclusão: embora as atitudes positivas dos enfermeiros especialistas em enfermagem de reabilitação face à morte, adquirissem um valor percentual superior, a diferença não foi significativa. Além disso, as atitudes negativas sinalizam para a necessidade de ser repensarem as estratégias de formação no contexto académico, mas também no contexto profissional.

Descritores: Atitude Frente à Morte; Morte; Enfermeiras Especialistas; Especialidades de Enfermagem; Enfermagem em Reabilitação; Hospitais.

\section{RESUMEN}

Objetivo: analizar las diferencias en el perfil de actitudes hacia la muerte entre enfermeros especializados en enfermería de rehabilitación y enfermeros especializados en otras áreas.

Método: estudio comparativo, cuantitativo, transversal, con la participación de 223 enfermeros especializadas de un hospital del norte de Portugal. Como instrumento de recolección de datos, se utilizó el cuestionario. Para el análisis de datos, se utilizaron estadísticas descriptivas y analíticas.

Resultados: en cuanto a las actitudes hacia la muerte, en los enfermeros especializados en enfermería de rehabilitación, predominaron las de enfoque, neutralidad, miedo, evitación y escape. En los enfermeros con experiencia en otras áreas, prevalecieron las actitudes de acercamiento, miedo, neutralidad, evitación y escape.

Conclusión: aunque las actitudes positivas de los enfermeros especializados en enfermería de rehabilitación hacia la muerte adquirieron un mayor valor porcentual, la diferencia no fue significativa. Además, las actitudes negativas señalan la necesidad de repensar las estrategias de aprendizaje en el contexto académico, pero también en el contexto profesional.

Descriptores: Actitud Frente a la Muerte; Muerte; Enfermeras Especialistas; Especialidades de Enfermería; Enfermería en Rehabilitación; Hospitales.

\section{ABSTRACT}

Objective: to analyse the differences in the profile of attitudes towards death between specialist nurses in rehabilitation nursing and specialist nurses in other areas. 
Method: comparative, quantitative, cross-sectional study, with the participation of 223 specialist nurses from a hospital in northern Portugal. A questionnaire was applied as a data collection instrument. For data analysis, descriptive and analytical statistics were used.

Results: as for attitudes towards death, in nurses who specialize in rehabilitation, those of approach, neutrality, fear, avoidance and escape predominated. In nurses with expertise in other areas, attitudes of approach, fear, neutrality, avoidance and escape prevailed.

Conclusion: although the positive attitudes towards death, of nurses specializing in rehabilitation, acquired a higher percentage value, the difference was not significant. In addition, negative attitudes signal the need to rethink training strategies in the academic, but also in the professional context.

Descriptors: Attitude to Death; Death; Nurse Specialists; Specialties, Nursing; Rehabilitation Nursing; Hospitals.

\section{INTRODUÇÃO}

Da mesma forma que o nascimento representa o início da vida, a morte é um episódio natural que marca o final da vida na terra ${ }^{(1-2)}$. O problema é que decorrente das inúmeras transformações que ocorreram ao longo da história ${ }^{(3)}$, o hospital passou a ser o local onde se morre e a morte é vista como um fracasso, marcado por sentimentos de impotência e frustração(4).

Num estudo epidemiológico nacional realizado em Portugal, constatou-se que em relação ao local de morte, de todos os óbitos registados num ano civil, $61,7 \%$ ocorreram em hospitais/clínicas e $29,6 \%$ no domicílio $^{(5)}$, dados que evidenciam a frequência com que os profissionais de saúde cuidam de pessoas em final de vida. Atendendo às particularidades do seu exercício profissional, os enfermeiros são os profissionais que passam mais tempo e em maior proximidade com as pessoas que vivenciam a morte e o processo de morrer, sendo que as suas atitudes podem constituir um aspeto facilitador ou dificultador do cuidado.

As atitudes acerca da morte podem ser classificadas como positivas ou negativas. Nas atitudes positivas destaca-se a aceitação da morte, enquanto no âmbito das atitudes negativas têm especial enfoque o medo e o evitamento da morte ${ }^{(2,6)}$. No que concerne à aceitação da morte, identificam-se três tipos de atitudes: aceitação neutral/neutralidade (caracterizada pelo entendimento da morte como uma parte integral da vida), aceitação como aproximação (caracterizada pela crença religiosa de uma vida feliz após a morte) e aceitação como escape (caracterizada pela aceitação da morte como o término de circunstâncias que provocam dor e sofrimento) $)^{(2,6)}$.

As investigações no âmbito das atitudes acerca da morte têm vindo a ser mais frequentes em diversas áreas científicas, por se acreditar que constituem componentes cruciais no processo de tomada de decisão(7). Os estudos realizados na área de enfermagem, apontam para o facto das atitudes dos enfermeiros frente à morte poderem influenciar a qualidade dos cuidados prestados às pessoas que vivenciam a morte e o processo de morrer ${ }^{(7)}$.

Embora vários autores salientem que a formação relativa à temática da morte e do processo de morrer culmine em atitudes mais positivas no cuidar de pessoas em fim de vida ${ }^{(2)}$, tem prevalecido a dúvida quanto à tipologia de formação mais relevante. Além disso, o facto da temática da morte e do processo de morrer ser abordada de forma superficial em todos os níveis de formação, tem sido frequentemente apontada pelos estudantes de enfermagem e pelos enfermeiros, como justificação para a falta de preparação que sentem na prestação de cuidados a pessoas em fim de vida ${ }^{(8-9)}$.

Durante a licenciatura em enfermagem, na sequência da estrutura curricular do curso, os estudantes percecionam fundamentalmente o seu papel no âmbito da promoção, da recuperação e da preservação da vida das pessoas doentes a quem prestarão cuidados. Perante tal enfoque, a morte começa a ser encarada como um imprevisto ou um fracasso ${ }^{(8)}$.

$\mathrm{Na}$ continuidade do percurso iniciado na licenciatura, os cursos de especialização em enfermagem, permitem aos enfermeiros qualificarem-se, tornando-os mais aptos para dar contributos tangíveis ao desenvolvimento da profissão e disciplina de enfermagem, bem como mais capacitados para dar resposta às diversas necessidades evidenciadas pelas pessoas ao longo do ciclo vital, onde inevitavelmente, se inclui a morte e o processo de morrer ${ }^{(10)}$.

Na sequência desta formação, independentemente das áreas de especialização, todos os enfermeiros especialistas partilham de um conjunto de competências comuns que se encontram distribuídas por quatro domínios: desenvolvimento de aprendizagens profissionais; gestão dos cuidados; melhoria da qualidade e responsabilidade profissional, ética e legal ${ }^{(10-11)}$.

Embora as atitudes dos enfermeiros especialistas acerca da morte possam influenciar a sua atuação perante pessoas a vivenciar a morte e o processo de morrer, importa também destacar o contributo que estes profissionais poderão dar, no seu contexto de trabalho, no âmbito de todo o processo de cuidar. De facto, na sequência do papel que desempenham nas equipas, nomeadamente no domínio da gestão dos cuidados, os enfermeiros especialistas podem ser determinantes para a qualidade dos cuidados que são prestados na fase final da vida. O acompanhamento e assessoria que podem e devem dar aos enfermeiros da sua equipa, bem com o papel de facilitador da sua aprendizagem, já previstos nas competências comuns $^{(11)}$, minimizarão o impacto provocado por algumas atitudes negativas dos profissionais perante a morte.

A verdade é que, cada vez mais frequente nas instituições hospitalares, a morte e o processo de morrer, exigem abordagens mais qualificadas ${ }^{(12)}$, onde 
para além da preparação técnico-científica, adquirem especial relevância as atitudes dos profissionais. Atendendo a que os conteúdos relativos à morte e ao processo de morrer são abordados de forma distinta nas diversas áreas de especialização, entende-se que conhecer o perfil de atitudes dos enfermeiros especialistas acerca da morte pode dar contributos para melhorar o processo formativo no âmbito da temática em estudo. Neste contexto, como pergunta de partida definiu-se: haverá diferenças no perfil de atitudes acerca da morte nos enfermeiros especialistas?

Perante o exposto, e atendendo à importância de estudar de forma faseada as atitudes dos enfermeiros das diferentes áreas de especialização, o objetivo deste estudo foi analisar as diferenças no perfil de atitudes acerca da morte entre enfermeiros especialistas em enfermagem de reabilitação e os enfermeiros especialistas em outras áreas.

\section{MÉTODO}

Estudo comparativo, quantitativo, descritivo e transversal, concretizado num hospital da região norte de Portugal. A população do estudo correspondeu aos enfermeiros especialistas a exercer funções na referida instituição hospitalar. A técnica de amostragem usada foi não probabilística de conveniência. Como critérios de inclusão foram definidos: enfermeiros especialistas a exercer funções nos serviços dos departamentos de medicina, cirurgia e medicina intensiva. Foram excluídos os enfermeiros que se encontravam de licença no momento da colheita de dados. A amostra ficou constituída por 223 enfermeiros especialistas, sendo que 99 desses enfermeiros são especialistas na área de enfermagem de reabilitação e 124 nas restantes áreas de especialização em enfermagem.

A colheita de dados foi realizada de fevereiro a março de 2018 e como instrumento de colheita de dados foi usado um questionário constituído por duas partes, a primeira referente à caracterização sociodemográfica dos enfermeiros especialistas e a segunda parte com a Escala de Avaliação do Perfil de Atitudes acerca da Morte $\left(\right.$ EAPAM) ${ }^{(6)}$. A EAPAM é composta por 32 itens distribuídos por cinco dimensões: medo (com 7 itens de avaliação), aceitação neutral/neutralidade (com 5 itens de avaliação), aceitação como aproximação (10 itens de avaliação), aceitação como escape (5 itens de avaliação) e evitamento (com 5 itens de avaliação). As respostas numa escala tipo Likert variam entre um (discordo completamente) e sete pontos (concordo completamente). 0 escore total pode oscilar entre $32 \mathrm{e}$ 224.

Para a análise dos dados recorreu-se à estatística descritiva e analítica, usando como recurso o programa Statistical Package for the Social Sciences (SPSS) versão 22.0. Atendendo ao objetivo do estudo, além das medidas de tendência central, utilizou-se o teste QuiQuadrado de Pearson e o Mann-Whitney U Test, considerando sempre um nível de significância de $5 \%$ $(p<0,05)$.

Importa referir que o estudo obteve parecer favorável da Comissão de Ética, com o número 102/2017, tendo sido aprovado em reunião do Conselho de Administração do hospital em estudo, em 30 de março de 2017. Em consonância com os princípios éticos, todos os participantes assinaram o consentimento informado, tendo sido garantida a confidencialidade e o anonimato.

\section{RESULTADOS}

Dos 223 participantes, 44,4\% possuíam a especialização em Enfermagem de Reabilitação e 55,6\% em outras áreas, sendo que desses $59,3 \%$ têm a especialização em Enfermagem Médico-Cirúrgica; 19,2\% a especialização em Enfermagem de Saúde Comunitária; 16,3\% a especialização em Enfermagem de Saúde Mental e Psiquiatria; 3,0\% a especialização em Enfermagem de Saúde Infantil e Pediatria; e 2,2\% a especialização em Enfermagem de Saúde Materna e Obstetrícia.

Nas Tabelas 1 e 2 apresentam-se os resultados relativos à caracterização sociodemográfica e profissional dos dois grupos em estudo: um grupo constituído pelos enfermeiros especialistas em enfermagem de reabilitação e outro grupo formado pelos enfermeiros com especialização em outras áreas de enfermagem.

Em relação às variáveis sociodemográficas (Tabela 1), e especificamente no que concerne ao sexo e à idade, com recurso estatístico ao Qui-quadrado de Pearson, confirmaram-se associações significativas $(p=0,021$ e $p=0,020$, respetivamente) entre os dois grupos de enfermeiros especialistas. Embora o sexo feminino fosse predominante nos dois grupos, importa destacar que no grupo de enfermeiros especialistas em enfermagem de reabilitação o número de enfermeiros do sexo masculino foi superior. Em relação à idade, nos enfermeiros especialistas em enfermagem de reabilitação prevaleceu a classe dos 36 aos 45 anos e nos enfermeiros com especialização em outras áreas a classe dos 25 aos 35 anos. Acresce ainda que os participantes dos dois grupos são maioritariamente casados e praticantes ou crentes em alguma religião.

No que se refere às variáveis profissionais (Tabela 2), com recurso estatístico ao Qui-quadrado de Pearson, constataram-se associações significativas no tempo de exercício profissional entre os dois grupos de enfermeiros especialistas $(p=0,038)$. Embora tivesse prevalecido nos dois grupos a classe entre os 11 e 20 anos, é de destacar que na classe de 1 até 10 anos a percentagem foi superior no grupo dos enfermeiros com especialização em outras áreas.

No que se refere ao serviço onde exercem funções, no grupo de enfermeiros especialistas em enfermagem de reabilitação prevaleceu a área médica e no grupo dos enfermeiros com especialização em outras áreas predominou a área intensiva. Quando questionados acerca da realização de formação no âmbito da morte, perda ou luto, apenas $4,0 \%$ dos participantes do grupo dos enfermeiros especialistas em enfermagem de reabilitação e 4,8\% dos enfermeiros com especialização em outra área, responderam afirmativamente. 


\begin{tabular}{|c|c|c|c|c|c|c|c|c|}
\hline \multirow[t]{2}{*}{ Variáveis } & \multirow[t]{2}{*}{ Indicadores } & \multicolumn{2}{|c|}{$\begin{array}{l}\text { Enfermeiros especialistas em } \\
\text { enfermagem de reabilitação }\end{array}$} & \multicolumn{2}{|c|}{$\begin{array}{l}\text { Enfermeiros especialistas } \\
\text { em outras áreas }\end{array}$} & \multicolumn{2}{|c|}{ Total } & \multirow[t]{2}{*}{ Sig.* } \\
\hline & & $\mathrm{n}$ & $\%$ & $\mathrm{n}$ & $\%$ & $\mathrm{n}$ & $\%$ & \\
\hline \multirow{2}{*}{ Sexo } & Fem. & 67 & 30,0 & 99 & 44,4 & 166 & 74,4 & \multirow{2}{*}{0,021} \\
\hline & Masc. & 32 & 14,3 & 25 & 11,2 & 57 & 25,6 & \\
\hline \multirow{5}{*}{ Idade } & $<25$ anos & 0 & 0,0 & 1 & 0,4 & 1 & 0,4 & \multirow{5}{*}{0,020} \\
\hline & 25 a 35 anos & 36 & 16,1 & 50 & 22,4 & 86 & 38,6 & \\
\hline & 36 a 45 anos & 56 & 25,1 & 49 & 22,0 & 105 & 47,1 & \\
\hline & 46 a 55 anos & 7 & 3,1 & 19 & 8,5 & 26 & 11,7 & \\
\hline & $>56$ anos & 0 & 0,0 & 5 & 2,2 & 5 & 2,2 & \\
\hline \multirow{4}{*}{ Estado civil } & Solteira/o & 34 & 15,2 & 51 & 22,9 & 85 & 38,1 & \multirow{4}{*}{0,573} \\
\hline & Casada/o & 60 & 26,9 & 66 & 29,6 & 126 & 56,5 & \\
\hline & Divorciada/o & 5 & 2,2 & 6 & 2,7 & 11 & 4,9 & \\
\hline & Viúva/o & 0 & 0,0 & 1 & 0,4 & 1 & 0,4 & \\
\hline \multirow{2}{*}{$\begin{array}{l}\text { É praticante ou crente } \\
\text { de alguma religião }\end{array}$} & Sim & 83 & 37,2 & 99 & 44,4 & 182 & 81,6 & \multirow{2}{*}{0,444} \\
\hline & Não & 16 & 7,2 & 25 & 11,2 & 41 & 18,4 & \\
\hline
\end{tabular}

Tabela 1 - Variáveis sociodemográficas dos dois grupos de enfermeiros especialistas | *Sig.: Significância-Qui-quadrado de Pearson

\begin{tabular}{|c|c|c|c|c|c|c|c|c|}
\hline \multirow[t]{2}{*}{ Variáveis } & \multirow[t]{2}{*}{ Indicadores } & \multicolumn{2}{|c|}{$\begin{array}{l}\text { Enfermeiros especialistas em } \\
\text { enfermagem de reabilitação }\end{array}$} & \multicolumn{2}{|c|}{$\begin{array}{c}\text { Enfermeiros especialistas } \\
\text { em outras áreas }\end{array}$} & \multicolumn{2}{|c|}{ Total } & \multirow[t]{2}{*}{ Sig.* } \\
\hline & & $\mathrm{n}$ & $\%$ & $\mathrm{n}$ & $\%$ & $\mathrm{n}$ & $\%$ & \\
\hline \multirow{4}{*}{$\begin{array}{l}\text { Tempo de exercício } \\
\text { profissional }\end{array}$} & 1 a 10 anos & 21 & 9,4 & 33 & 14,8 & 54 & 24,2 & \multirow{4}{*}{$0,03 \varepsilon$} \\
\hline & 11 a 20 anos & 57 & 25,6 & 53 & 23,8 & 110 & 49,3 & \\
\hline & 21 a 30 anos & 21 & 9,4 & 32 & 14,3 & 53 & 23,8 & \\
\hline & $>31$ anos & 0 & 0,0 & 6 & 2,7 & 6 & 2,7 & \\
\hline \multirow{3}{*}{ Serviço } & Área médica & 38 & 17,0 & 37 & 16,6 & 75 & 33,6 & \multirow{3}{*}{0,128} \\
\hline & Área cirúrgica & 26 & 11,7 & 23 & 10,3 & 49 & 22,0 & \\
\hline & Área intensiva & 35 & 15,7 & 64 & 28,7 & 99 & 44,4 & \\
\hline \multirow{2}{*}{$\begin{array}{l}\text { No último ano fez } \\
\text { alguma formação sobre } \\
\text { morte, perda ou luto }\end{array}$} & Sim & 4 & 1,8 & 6 & 2,7 & 10 & 4,5 & \multirow{2}{*}{0,765} \\
\hline & Não & 95 & 42,8 & 117 & 52,7 & 212 & 95,5 & \\
\hline
\end{tabular}

Tabela 2 - Variáveis profissionais dos dois grupos de enfermeiros especialistas | *Sig.: Significância-Qui-quadrado de Pearson

Itens da Escala

Sig.*

1. A morte é sem dúvida uma experiência cruel

0,517

2. A perspetiva da minha própria morte provoca-me ansiedade

0,981

3. Evito a todo o custo pensamentos relacionados com a 0,573 morte

4. Acredito que depois de morrer irei para o céu $\quad 0,806$

5. A morte traz um fim para todos os meus problemas 0,616

6. A morte deve ser vista como um acontecimento 0,878
natural, inegável e inevitável natural, inegável e inevitável

7. Incomodo-me com a finalidade da morte $\quad 0,048$

8. A morte é a entrada num lugar último de satisfação $\quad 0,012$

9. A morte providencia um escape para este mundo 0,407 terrível

10. Sempre que um pensamento relacionado com a 0,674 morte me vem à cabeça tento afastá-lo a todo o custo

11. A morte é a libertação da dor e do sofrimento $\quad 0,141$

\begin{tabular}{l|l} 
12. Tento sempre não pensar na morte & 0,647
\end{tabular}

13. Acredito que o céu será um lugar muito melhor do 0,403 que este mundo

14. A morte é um aspeto natural da vida $\quad 0,658$

15. A morte é a união com Deus e a felicidade eterna $\quad 0,077$

16. A morte traz a promessa de uma vida nova e gloriosa 0,237

17. Eu não temo a morte nem a desejo $\quad 0,668$

\begin{tabular}{l|l}
\hline 18. Tenho um intenso medo da morte & 0,278
\end{tabular}

19. Evito a todo o custo pensar acerca da morte $\quad 0,213$
Itens da Escala

Sig.*

20. O assunto da vida depois da morte perturba-me 0,335 muito

21. Assusta-me o facto de a morte significar o fim de tudo o que eu conheço

22. Olho para o futuro, depois da morte, como a reunião com as pessoas que amei

0,690

23. Vejo a morte como um alívio para o sofrimento terreno

24. A morte é simplesmente uma parte do processo da vida

0,526

25. Vejo a morte como uma passagem para um lugar eterno e santo

26. Tento não fazer nada que esteja relacionado com o assunto da morte

27. A morte oferece uma maravilhosa libertação da alma 0,389

28. Uma coisa que me dá conforto face à morte são as minhas crenças

29. Vejo a morte como alívio do fardo desta vida 0,636

30. A morte não é boa nem má $\quad 0,634$

31. Olho antecipadamente para a vida depois da morte 0,149

32. A incerteza de nada saber ao que acontece depois da morte

*Sig.: Significância - Qui-quadrado de Pearson

Tabela 3 - Análise da significância das atitudes acerca da morte nos dois grupos de enfermeiros especialistas 
Na análise das atitudes acerca da morte (Tabela 3), confirmaram-se, com recurso estatístico ao Quiquadrado de Pearson, associações significativas nos itens 7 e 8 da Escala. Neste sentido, a atitude "Incomodo-me com a finalidade da morte" ( $p=0,048)$, incluída na dimensão medo, foi mais predominante no grupo dos enfermeiros com especialização em outras áreas. Por outro lado, a atitude "A morte é a entrada num lugar último de satisfação” ( $p=0,012)$, incluída na dimensão aceitação como aproximação, foi mais prevalente no grupo dos enfermeiros especialistas em enfermagem de reabilitação.

Decorrente da análise às dimensões que integram as diversas atitudes acerca da morte (Tabela 4), nos enfermeiros especialistas em enfermagem de reabilitação predominaram as de aproximação $(37,30$ pontos), seguidas das de neutralidade (27,89 pontos), medo (26,51 pontos), evitamento (17,88 pontos) e escape (15,04 pontos). No caso dos enfermeiros com especialização em outras áreas prevaleceram as de aproximação (36,38 pontos), seguidas das de medo (27,78 pontos), neutralidade (27,30 pontos), evitamento $(17,67$ pontos) e escape $(14,75)$.

\begin{tabular}{|c|c|c|c|}
\hline $\begin{array}{l}\text { Dimensões das } \\
\text { atitudes face à morte }\end{array}$ & $\begin{array}{c}\text { Área de } \\
\text { especialização }\end{array}$ & Média & $\begin{array}{l}\text { Desvio- } \\
\text {-padrão }\end{array}$ \\
\hline \multirow{2}{*}{ Medo } & $\begin{array}{l}\text { Enfermagem de } \\
\text { Reabilitação }\end{array}$ & 26,51 & 8,902 \\
\hline & $\begin{array}{l}\text { Outras áreas de } \\
\text { especialização }\end{array}$ & 27,78 & 8,305 \\
\hline \multirow{2}{*}{ Evitamento } & $\begin{array}{l}\text { Enfermagem de } \\
\text { Reabilitação }\end{array}$ & 17,88 & 7,718 \\
\hline & $\begin{array}{l}\text { Outras áreas de } \\
\text { especialização }\end{array}$ & 17,67 & 7,266 \\
\hline \multirow{2}{*}{$\begin{array}{c}\text { Aceitação } \\
\text { Neutral/Neutralidade }\end{array}$} & $\begin{array}{l}\text { Enfermagem de } \\
\text { Reabilitação }\end{array}$ & 27,89 & 3,682 \\
\hline & $\begin{array}{l}\text { Outras áreas de } \\
\text { especialização }\end{array}$ & 27,30 & 4,089 \\
\hline \multirow{2}{*}{$\begin{array}{l}\text { Aceitação como } \\
\text { Aproximação }\end{array}$} & $\begin{array}{l}\text { Enfermagem de } \\
\text { Reabilitação }\end{array}$ & 37,30 & 9,884 \\
\hline & $\begin{array}{l}\text { Outras áreas de } \\
\text { especialização }\end{array}$ & 36,38 & 12,600 \\
\hline \multirow{2}{*}{$\begin{array}{l}\text { Aceitação como } \\
\text { Escape }\end{array}$} & $\begin{array}{l}\text { Enfermagem de } \\
\text { Reabilitação }\end{array}$ & 15,04 & 5,604 \\
\hline & $\begin{array}{l}\text { Outras áreas de } \\
\text { especialização }\end{array}$ & 14,75 & 6,303 \\
\hline
\end{tabular}

Tabela 4 - Escores médios das atitudes acerca da morte, nos dois grupos de enfermeiros especialistas

Ainda que existam algumas diferenças nas médias das atitudes acerca da morte, ao avaliar as dimensões e a escala global com recurso ao Test $U$ de Mann-Whitney de amostras independentes, foi possível confirmar que essas diferenças entre os dois grupos de enfermeiros especialistas não são significativas (Tabela 5).

\section{Hipóteses nulas}

Sig.*

A distribuição da atitude: medo é a mesma entre o grupo dos enfermeiros especialistas em enfermagem de reabilitação e o grupo dos 0,288 enfermeiros especialistas em outras áreas

A distribuição da atitude: evitamento é a mesma entre o grupo dos enfermeiros especialistas em enfermagem de reabilitação e o grupo dos 0,929 enfermeiros especialistas em outras áreas

A distribuição da atitude: neutralidade é a mesma entre o grupo dos enfermeiros especialistas em enfermagem de reabilitação e o grupo dos 0,520 enfermeiros especialistas em outras áreas

A distribuição da atitude: aproximação é a mesma entre o grupo dos enfermeiros especialistas em enfermagem de reabilitação e o grupo dos 0,606 enfermeiros especialistas em outras áreas

A distribuição da atitude: escape é a mesma entre o grupo dos enfermeiros especialistas em
enfermagem de reabilitação e o grupo dos enfermeiros especialistas em outras áreas

A distribuição do total da escala é a mesma entre - grupo dos enfermeiros especialistas em enfermagem de reabilitação e o grupo dos 0,923 enfermeiros especialistas em outras áreas

Tabela 5 - Análise da significância das dimensões das atitudes acerca da morte nos dois grupos de enfermeiros especialistas *Sig.: Significância - Test $U$ de Mann-Whitney de amostras independentes

\section{DISCUSSÃO}

Embora todos estejam consciencializados de que a morte é um facto inevitável, continua a ser difícil aceitá-la(8). $\mathrm{Na}$ sequência da sua crescente institucionalização, a convivência com a morte e o processo de morrer é uma realidade com que se deparam diariamente os profissionais da área da saúde, nomeadamente os que exercem a sua atividade profissional no contexto hospitalar ${ }^{(12)}$, de que são exemplo neste estudo, os enfermeiros especialistas. Embora a morte seja um acontecimento natural da vida humana, vários autores têm refletido sobre a importância de identificar as atitudes dos profissionais de saúde acerca da morte, uma vez que as mesmas podem influenciar a forma de cuidar das pessoas que se encontram na fase final da vida(2). Atendendo às especificidades do seu exercício profissional, os enfermeiros constituem os profissionais cujas atitudes têm vindo a ser mais profundamente pesquisadas.

Para além disso, e como acrescentam alguns autores, perante a resistência natural das equipas de saúde frente à morte e ao processo de morrer, são os enfermeiros que frequentemente assumem o papel de líder $^{(4,13)}$, o que de facto reforça ainda mais o contributo que poderão dar na prática assistencial diante da morte e do processo de morrer.

Neste estudo, analisar o perfil de atitudes de enfermeiros especialistas constituiu um enorme desafio na medida em que o impacto das atitudes destes enfermeiros pode ser significativo no cuidado que prestam às pessoas, mas também na influência que podem ter na atuação dos enfermeiros de cuidados 
gerais do serviço onde se encontram(11), o que consequentemente terá uma repercussão favorável na qualidade dos cuidados que são prestados às pessoas a vivenciar a morte e o processo de morrer.

Numa investigação realizada com enfermeiros especialistas, os autores confirmaram que os participantes estavam consciencializados das mudanças que ocorrerão no seu exercício profissional após terminarem a especialização, nomeadamente mais segurança na tomada de decisão, bem como maior capacidade para a visão integral da pessoa e individualização das respetivas necessidades ${ }^{(10)}$, aspeto que adquire especial relevância na assistência prestada às pessoas que vivenciam a morte e o processo de morrer ${ }^{(9)}$.

O mencionado torna ainda mais relevante este estudo, que conta com a participação exclusiva de enfermeiros especialistas. Em relação às características sociodemográficas destes participantes, verificámos que nos dois grupos de enfermeiros especialistas prevaleceu o sexo feminino e o estado civil de casado. Embora o sexo feminino fosse predominante, destacouse o facto de no grupo de enfermeiros especialistas em enfermagem de reabilitação, o número de enfermeiros do sexo masculino ter sido superior ao do outro grupo de participantes, o que corrobora os dados publicados pela Ordem dos Enfermeiros ${ }^{(14)}$. Em relação à idade, nos enfermeiros especialistas em enfermagem de reabilitação predominou a classe dos 36 aos 45 anos, e no grupo de enfermeiros com especialização em outras áreas teve percentual idêntico a classe dos 25 aos 35 anos e dos 36 aos 45 anos $^{(14)}$. Esta diferença pode ser justificada pelo crescente número de enfermeiros que nos últimos anos têm frequentado, no início do seu exercício profissional, cursos de especialização de enfermagem em outras áreas, além da enfermagem de reabilitação.

No que se refere às características profissionais, os enfermeiros especialistas em enfermagem de reabilitação exercem a sua atividade profissional predominantemente nos serviços da área médica, por outro lado, nos enfermeiros com especialização em outras áreas o percentual foi mais elevado nos serviços da área intensiva. O facto de no grupo de enfermeiros com especialização em outras áreas, o número de especialistas em enfermagem médico-cirúrgica ser o mais elevado, justifica esse maior percentual nos serviços da área intensiva, contextos que concentram frequentemente elevado número de especialistas nesta área. Relativamente ao tempo de exercício profissional, nos dois grupos prevaleceu a classe entre os 11 e 20 anos, sendo de destacar que na classe de 1 até 10 anos a percentagem foi superior no grupo dos enfermeiros com especialização em outras áreas.

A análise dos escores das atitudes acerca da morte revelou que nos dois grupos foi predominante a dimensão aceitação como aproximação, que sustentada na religião, compreende a ideia de uma vida feliz após a morte. Conforme referido por alguns autores, é como se o profissional de saúde, com base nas suas conceções religiosas, desejasse uma intercessão divina ${ }^{(15)}$. $\mathrm{Na}$ maior parte dos estudos realizados com enfermeiros ou estudantes de enfermagem, a dimensão que prevalece é a aceitação neutral/neutralidade ${ }^{(2,7)}$ e não a aceitação como aproximação. Embora seja consensual que a crença em alguma religião, para além de dar sentido à vida e à morte, ajuda os profissionais e as pessoas doentes a confrontarem-se com a morte e o processo de morrer ${ }^{(9)}$, vários autores consideram de maior relevância a aceitação neutral. $\mathrm{Na}$ sua perspetiva, a dimensão aceitação neutral/neutralidade é potenciadora de uma atuação profissional que procura dar resposta mais efetiva às diversas necessidades manifestadas pelas pessoas a vivenciar a morte e o processo de morrer, respeitando simultaneamente o direito dessas pessoas participarem nas decisões sobre os cuidados, assim como em aceitar ou rejeitar esses mesmos cuidados ${ }^{(12,16)}$.

Neste seguimento, e retomando os resultados deste estudo, em segundo lugar, no grupo dos enfermeiros especialistas em enfermagem de reabilitação prevaleceu a aceitação neutral/neutralidade, caracterizada pelo entendimento da morte como um fenómeno natural da vida, seguida pela dimensão medo da morte. No caso dos enfermeiros com especialização em outras áreas, após a dimensão aceitação como aproximação, prevaleceu o medo da morte, seguido da aceitação neutral/neutralidade. Na visão de alguns autores embora o medo da morte seja uma estratégia de defesa do ego contra o sofrimento, pode dificultar a compreensão sobre o momento vivido, bem como sobre o próprio significado da vida(2). Estudos apontam que em relação às atitudes frente à morte, quanto menor a idade, maior é o escore na dimensão medo(2), o que efetivamente pode ser um dos fatores que justifica as diferenças percentuais encontradas nos dois grupos em estudo. Além disso, um menor tempo de exercício profissional e, consequentemente, um menor tempo de experiência, verificado num maior número de participantes do grupo dos enfermeiros com especialização em outras áreas, pode tornar mais evidente o medo da morte. Isto porque, conforme mencionado por alguns autores, a experiência na prática assistencial vai ajudando gradualmente a enfrentar o medo da morte ${ }^{(9,17)}$.

Face ao mencionado anteriormente, será que os enfermeiros têm de esperar que os anos de experiência os ajudem a lidar com a morte? De facto, os resultados obtidos em relação à dimensão medo da morte, impõe a necessidade de se refletir sobre o modo como estes profissionais têm sido preparados para enfrentar a inevitabilidade da morte. Embora seja indiscutível que face à evolução técnico-científica na área da saúde, a formação especializada dote os enfermeiros de um nível de conhecimento acrescido e promova o desenvolvimento de competências imprescindíveis à prestação de cuidados a pessoas em condições clínicas cada vez mais complexas ${ }^{(18)}$, o mesmo poderá não estar a acontecer perante pessoas em situação de fim de vida. Neste contexto, importa resgatar o referido por uma teórica de enfermagem, que recentemente advertiu para o facto das tecnologias e das políticas de medicalização existentes nos hospitais estarem a desviar a enfermagem das suas bases disciplinares, correndo o risco de sob pressão vir a sujeitar-se a uma visão medicalizada e curativista das experiências vividas pelas pessoas ${ }^{(19)}$. Face a esta possibilidade, é 
emergente que a atuação dos enfermeiros especialistas perante pessoas que vivenciam a morte e o processo de morrer seja congruente com os referenciais teóricos da disciplina, nomeadamente com os que evidenciam potencial para sustentar a prática destes profissionais, o que de facto, nem sempre se tem constatado ${ }^{(12)}$.

Ainda que não se tivessem confirmado diferenças significativas entre o perfil de atitudes dos dois grupos de enfermeiros especialistas, importa salientar o escore mais elevado na dimensão aceitação neutral/neutralidade no caso dos enfermeiros especialistas em enfermagem de reabilitação, uma vez que esta atitude é de forma consensual vista como um ponto positivo, indicando que estes enfermeiros aceitam a morte como parte integrante da vida ${ }^{(7)}$. Atendendo a que no âmbito das suas competências específicas, os enfermeiros especialistas em enfermagem de reabilitação cuidam de pessoas com necessidades especiais, ao longo de todo o ciclo vital $^{(20)}$, torna-se ainda mais relevante o entendimento da morte como algo natural. De facto, a aceitação neutral/neutralidade potenciará uma atuação do enfermeiro especialista em enfermagem de reabilitação não apenas centrada do domínio da função e no controlo de sinais e sintomas, ainda muito evidente ${ }^{(21)}$, mas também nos aspetos psicoemocionais e sociais que interferem na qualidade de vida e no bemestar da pessoa ${ }^{(20)}$, que vivencia a morte e o processo de morrer. $O$ enfoque do enfermeiro especialista em enfermagem de reabilitação não passa nestas situações por reabilitar para a vida, mas sim habilitar para a morte, não no sentido de a antecipar, mas sim no sentido de tornar a pessoa hábil para viver este período com a máxima qualidade possível(22). 0 problema é que, o referido dificilmente será possível se o enfermeiro especialista em enfermagem de reabilitação manifestar acerca da morte atitudes pouco facilitadoras dessa atuação.

No que se refere às restantes dimensões da EAPAM, nos dois grupos obtiveram escores mais baixos a dimensão evitamento, seguida da dimensão aceitação como escape. Na perspetiva dos autores, face ao evitamento, é importante não esquecer que os profissionais de saúde também são humanos e, portanto, sofrem com as questões relativas à morte e para além disso, a maioria desses profissionais não foi preparada pela sociedade, nem pela formação académica para lidar com a morte ${ }^{(13)}$. Acresce ainda que não estando preparados, é compreensível a adoção de atitudes de escape perante a morte.

De facto, no que se refere à preparação dos enfermeiros para lidar com a morte e o processo de morrer são conhecidas as fragilidades que atualmente existem na formação académica $^{(9)}$. A vivência de sentimentos negativos acerca da morte, está frequentemente atrelada a uma formação voltada para a recuperação e para a cura e negligente na discussão sobre a finitude da vida ${ }^{(4)}$. Esta lacuna nas matrizes curriculares, dos cursos de licenciatura e de póslicenciatura, tem culminado na dificuldade dos enfermeiros e enfermeiros especialistas em lidarem com a temática da morte e do processo de morrer ${ }^{(2,8,15,23)}$, o que consequentemente influencia a qualidade dos cuidados prestados. Acresce a isso, o facto de mais de $95 \%$ dos participantes referirem não terem frequentado, no último ano, formação sobre a morte, o morrer, a perda ou o luto.

Embora vários autores já tenham abordado as atitudes dos enfermeiros frente à morte ${ }^{(4,9)}$, a inexistência de estudos sobre as atitudes de enfermeiros especialistas dificultou a discussão dos resultados obtidos. Mesmo assumindo como limitação o facto de o estudo ter sido realizado em apenas um contexto hospitalar, considerase que o mesmo traz contribuições que advertem para a necessidade de se adotarem estratégias que favoreçam a atuação dos enfermeiros especialistas perante as pessoas em fase final da vida, perante os colegas da equipa, mas que simultaneamente, minimizem o seu sofrimento em lidar com a morte e o processo de morrer. Neste contexto, a par da formação e do acompanhamento dos profissionais, têm sido enaltecidos os momentos de partilha de sentimentos e de vivências entre os diferentes elementos da equipa, que para além de promoverem suporte psicoemocional, serão um contributo para a normalização da morte como algo natural ${ }^{(4,9)}$.

\section{CONCLUSÃO}

Os resultados obtidos neste estudo vêm reforçar a necessidade de se investir na formação sobre a temática da morte e do morrer não só no curso de licenciatura, mas também ao nível da formação pósgraduada, de que são exemplo os cursos de especialização. A presença de atitudes negativas face à morte, com especial enfoque o medo, para além de poder influenciar a atuação dos enfermeiros especialistas perante pessoas a vivenciar a morte e o processo de morrer, podem acarretar um sofrimento pessoal que importa minimizar.

Embora as atitudes positivas dos enfermeiros especialistas em enfermagem de reabilitação acerca da morte, adquirissem um valor percentual superior, a diferença não foi significativa, e para além disso, as atitudes negativas são ainda notórias. Neste sentido, a par da reformulação dos conteúdos incluídos nos cursos de licenciatura e pós-licenciatura, é importante no contexto hospitalar (re)pensar a formação contínua no âmbito desta temática, bem como desenvolver estratégias que garantam o acompanhamento dos profissionais que se deparem com dificuldades em lidar com a morte e o processo de morrer.

\section{REFERÊNCIAS BIBLIOGRÁFICAS}

1. Cardoso F, Silva M, Alves C, Martins M. The dying process: its manifestation in nursing records. Rev Enf Ref. [serial on the Internet]. 2019 [cited 2020 Apr 13]; IV(21);121-130. Available from:

http://www.scielo.mec.pt/pdf/ref/vserIVn21/serIVn21a12.pdf

2. Souza MCS, Sousa JM, Lago DMSK, Borges MS, Ribeiro LM, Guilhem DB. Evaluation of the Death Attitude Profile-Revised: a study with health science undergraduate students. Texto Contexto Enferm. [serial on the Internet]. 2017 [cited 2020 Apr 12]; 26(4): e3640016. Available from: http://www.scielo.br/pdf/tce/v26n4/en_0104-0707-tce-26-04e3640016.pdf 
3. Silva RS, Pereira A, Mussi FC. Comfort for a good death: perspective nursing staff's of intensive care. Esc Anna Nery [serial on the Internet]. 2015 [cited $2020 \mathrm{Apr} 14$ ]; 19(1):40-46. Available from: http://www.scielo.br/pdf/ean/v19n1/en_1414-8145-ean19-01-0040.pdf

4. Bastos RA, Lamb FA, Quintana AM, Beck CL, Carnevale F. The experiences of nurses in the dying process: a qualitative metasynthesis. Revista Portuguesa de Enfermagem de Saúde Mental [serial on the Internet]. 2017 [cited 2020 Apr 04];(17):58-64. Available http://www.scielo.mec.pt/pdf/rpesm/n17/n17a08.pdf

5. Gomes B, Sarmento VP, Ferreira PL, Higginson IJ. Epidemiological study of place od death in Portugal in 2010 and comparison with the preferences of the portuguese population. Acta Med Port. [serial on the Internet]. 2013 [cited 2020 Apr 07];26(4):327-334. Available

https://www.ncbi.nlm.nih.gov/pubmed/24016640

from:

6. Loureiro LMJ. Translation and adaptation of the Revised Death Attitude Profile (DAP-R). Rev Enf Ref. [serial on the Internet]. 2010 [cited $2020 \mathrm{Apr}$ 10]; III(1):101-108. Available from: http://www.scielo.mec.pt/pdf/ref/vserllln1/serllln1a11.pdf

7. Machado RS, Oriá MOB, Fernandes MA, Gouveia MTO, Silva GRF. Translation and cultural adaptation of Death Attitude Profile Revised (DAP-R) for use in Brasil. Texto Contexto Enferm. [serial on the Internet]. 2019 [cited $2020 \mathrm{Apr}$ 09]; 28: e20180238. Available from: http://www.scielo.br/pdf/tce/v28/1980-265Xtce-28-e20170336.pdf

8. Ivo OP, Pedroso KO. The Process of Death and Dying: A Vision of Nursing Students. Rev. Psic. [serial on the Internet]. 2017 [cited 2020 Apr 19]; 11(34):305-321. Available from: http://docplayer.com.br/46512009-The-process-of-death-anddying-in-nurses-vision.html

9. Salum MEG, Kahl C, Cunha KS, Koerich C, Santos TO, Erdmann AL. The process of death and dying: challenges in nursing care for patients and family members. Rev Rene [serial on the Internet]. 2017 [cited 2020 Apr 21];18(4):528-35. Available from: http://periodicos.ufc.br/rene/article/view/20281/30815

10. Silva RMO, Luz MDA, Fernandes JD, Silva LS, Cordeiro ALAO, Mota LSR. Becoming a specialist: portuguese nurses 'expectations after completing the specialization program. Rev Enf Ref. [serial on the Internet]. 2018 [cited 2020 Apr 20];IV(16):147-154. Available from:

http://www.scielo.mec.pt/pdf/ref/vserlVn16/serIVn16a15.pdf

11. Portugal. Ministério da Saúde. Regulamento n. ${ }^{\circ} 140 / 2019$, de 6 de fevereiro de 2019. Regulamento das Competências Comuns do Enfermeiro Especialista. Diário da República. 2019 fev; 2. a série. p 4744-4750. Available from: https: / / www.ordemenfermeiros.pt/media/10778/0474404750.p df

12. Cardoso MFPT, Ribeiro OMPL, Martins MMFPS. Death and dying: contributions to a practice based on nursing theoretical frameworks. Rev. Gaúcha Enfermagem. [serial on the Internet]. 2019 [cited 2020 Apr 20];40: e20180139. Available from: http://www.scielo.br/pdf/rgenf/v40/en_1983-1447-rgenf-40e20180139.pdf

13. Prado RT, Leite JL, Silva íR, Silva LJ. Communication in the management of the nursing care before the death and dying process. Texto Contexto Enferm. [serial on the Internet]. 2019 [cited 2020 Apr 08]; 28:e20170336. Available from: http://www. scielo.br/pdf/tce/v28/1980-265X-tce-28e20170336.pdf

14. Ordem dos Enfermeiros. Estatística dos Enfermeiros Especialistas 2017. Lisboa: Ordem dos Enfermeiros; 2017 Dez. Available from: https://www.ordemenfermeiros.pt/media/11129/2017_dadoses tatisticos_especialistas.pdf

15. Praxedes AM, Araújo JL, Nascimento EGC. A morte e o morrer no processo de formação do enfermeiro. Psic., Saúde \& Doenças [serial on the Internet]. 2018 [cited 2020 Apr 20]; 19(2): 369-376. Available http://www.scielo.mec.pt/pdf/psd/v19n2/v19n2a16.pdf

16. McEwen M, Wills EM. Bases teóricas de enfermagem. 4 ed. Porto Alegre: Artmed; 2016

17. Gonçalves LHT, Polaro SHI, Feitosa ES, Rodrigues ARS, Monteiro HK. Teach nursing care about people in the end of life experience report. Rev Enferm UFPE on line [serial on the Internet]. 2013 [cited 2020 Apr 11];7(10):6047-6053. Available from:

https://periodicos.ufpe.br/revistas/revistaenfermagem/article/ view/12234/14842

18. Silva R, Lage I, Macedo E. Vivências dos enfermeiros sobre morte e morrer em cuidados intensivos: Uma reflexão fenomenológica. Revista Portuguesa de Enfermagem de Saúde Mental [serial on the Internet]. 2018 [cited $2020 \mathrm{Apr}$ 17];(20):34-42. Available from: http://www.scielo.mec.pt/pdf/rpesm/n20/n20a05.pdf

19. Watson J. Clarifying the discipline of nursing as foundational to development of professional nursing. Texto Contexto Enferm. [serial on the Internet]. 2017 [cited 2020 Apr 21]; 26(4): editorial. Available from: http://www.scielo.br/pdf/tce/v26n4/en_01040707-tce-26-04-0000.pdf

20. Portugal. Ministério da Saúde. Regulamento n. ${ }^{\circ} 392 / 2019$, de 03 de maio de 2019. Regulamento das Competências Específicas do Enfermeiro Especialista em Enfermagem de Reabilitação. Diário da República. 2019 maio; 2. ${ }^{a}$ série. p 13565-13568. Available from: https://dre.pt/web/guest/pesquisa//search/122216893/details/normal?l=1

21. Cardoso MF, Martins MM, Ribeiro 0 . Nursing care documentation during the dying process - How do the rehabilitation nurses difer? Revista Portuguesa de Enfermagem de Reabilitação [serial on the Internet]. 2019 [cited $2020 \mathrm{Apr}$ 04];2(2):33-40. Available from: https://www.aper.pt/ficheiros/revista/RPERv2n2.pdf

22. Santos AS, Pêla J. Reabilitação da Pessoa em Cuidados Paliativos e Fim de Vida. In Marques-Vieira C, Sousa L. Cuidados de Enfermagem de Reabilitação à Pessoa ao Longo da Vida. Loures: Lusodidacta; 2017. (p. 605-618).

23. Nunes EC, Santos AD. Challenges of nursing teaching-learning to care for human dying - professors' perceptions. Esc Anna Nery [serial on the Internet]. 2017 [cited $2020 \mathrm{Apr}$ 06];21(4): e20170091. Available from: http://www.scielo.br/pdf/ean/v21n4/1414-8145-ean-21779465-EAN-2017-0091.pdf 